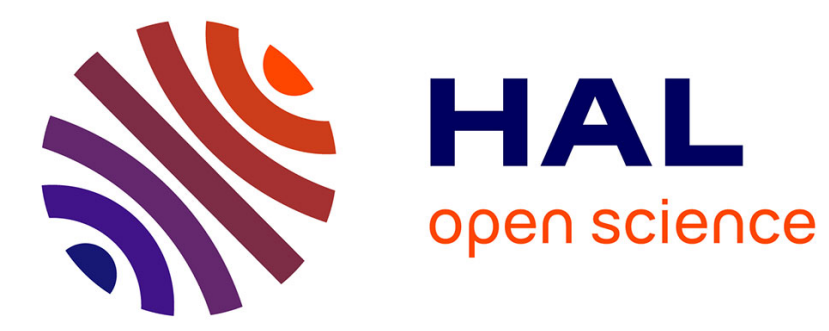

\title{
Possibilités d'obtention et intérêt des génotypes maigres en aviculture
}

\author{
B. Leclercq
}

\section{To cite this version:}

B. Leclercq. Possibilités d'obtention et intérêt des génotypes maigres en aviculture. Productions Animales, 1989, 2 (4), pp.275-286. hal-00895875

\section{HAL Id: hal-00895875 \\ https://hal.science/hal-00895875}

Submitted on 1 Jan 1989

HAL is a multi-disciplinary open access archive for the deposit and dissemination of scientific research documents, whether they are published or not. The documents may come from teaching and research institutions in France or abroad, or from public or private research centers.
L'archive ouverte pluridisciplinaire HAL, est destinée au dépôt et à la diffusion de documents scientifiques de niveau recherche, publiés ou non, émanant des établissements d'enseignement et de recherche français ou étrangers, des laboratoires publics ou privés. 
INRA Prod. Anim., 1989, 2 (4), 275-286

\section{B. LECLERCQ}

INRA Station de Recherches avicoles Nouzilly 37380 Monnaie

\section{Possibilités d'obtention et intérêt des génotypes maigres en aviculture}

Considérées comme des sources de viande maigre, les espèces avicoles n'ont fait l'objet jusqu'à présent d'aucune sélection particulière contre l'état d'adiposité. Les croisements commerciaux actuels, fruits d'une sélection intense et exclusive sur la vitesse de croissance, produisent des carcasses de plus en plus grasses. Les sélections expérimentales entreprises pour la production de lignées maigres ont conclu à des avantages substantiels. Les nouveaux génotypes peuvent représenter un progrès significatif des filières avicoles de demain.

L'aviculture est demeurée très longtemps la seule production de viande dont les produits étaient commercialisés entiers, sans subir aucune transformation industrielle après abattage. C'est aussi la production où les progrès de la génétique ont été les plus spectaculaires. La petite taille des espèces, leur prolificité, le faible écart entre générations et la concentration de l'activité de sélection au sein de quelques firmes privées sont autant de facteurs qui ont largement favorisé le progrès génétique. Celuici, essentiellement basé sur l'amélioration de la vitesse de croissance, s'est peu à peu accompagné de caractères défavorables notamment les troubles locomoteurs et l'excès d'adiposité. Or parallèlement l'industrie avicole s'est orientée de plus en plus vers la transformation de ses produits; en particulier vers la découpe et la

\section{Résumé}

La sélection pratiquée en aviculture sur la vitesse de croissance a conduit à la production d'animaux de plus en plus gras. Or l'importance des dépôts adipeux est un caractère à héritabilité élevée, ce qui autorise une sélection efficace vers la maigreur. Les sélections expérimentales réalisées jusqu'à présent utilisent comme paramètre soit l'efficacité alimentaire, soit les lipoprotéines plasmatiques de très basse densité soit l'importance du tissu adipeux abdominal mesuré sur collatéraux après abattage. Ces différentes méthodes ont permis d'obtenir aisément des génotypes maigres. Ceux-ci présentent toujours un indice de consommation plus faible, une meilleure efficacité de la transformation des protéines alimentaires en protéines corporelles, un meilleur rendement en carcasse et une plus forte proportion de muscles. L'orientation de la sélection de toutes les espèces avicoles vers une amélioration des qualités de carcasse et en particulier la réduction de l'état d'engraissement peut représenter un progrès notable en aviculture. vente des carcasses en morceaux (cuisses, filets, etc). La composition corporelle est donc devenue une préoccupation de plus en plus importante chez toutes les espèces (poulet, canard, pintade et, dans une moindre mesure, le dindonneau). Longtemps les moyens de lutte contre l'engraissement ont été d'ordre nutritionnel. On peut affirmer sans exagération qu'aujourd'hui l'aviculture gaspille une partie des protéines alimentaires (en particulier le soja d'importation) à la seule fin de faire maigrir les animaux et d'abaisser l'indice de consommation.

C'est pourquoi l'approche génétique est apparue de plus en plus urgente. Presque simultanément plusieurs sélections expérimentales ont été entreprises dans plusieurs laboratoires européens afin d'explorer cette voie et d'en évaluer les avantages. Nous rapportons dans cet article les principales acquisitions réalisées à cette occasion et nous tentons d'en dégager quelques éléments de prospective pour la filière avicole.

\section{La répartition des lipides de réserve}

Les viandes de volailles ont la réputation d'être des produits pauvres en lipides; c'est tout particulièrement le cas du poulet, de la dinde et de la pintade. Toutefois toutes les espèces avicoles possèdent des tissus adipeux plus ou moins abondants et répartis différemment selon les espèces. Le canard présente des 
Tableau 1. Teneurs en lipides totaux et proportions de gras abdominal (exprimées par rapport au poids vif) des espèces d'intérêt avicole à l'âge d'abattage.

\begin{tabular}{|l|c|c|}
\hline & $\begin{array}{c}\text { Lipides totaux } \\
\text { g/kg }\end{array}$ & $\begin{array}{c}\text { Gras abdominal } \\
\text { g/kg }\end{array}$ \\
\hline $\begin{array}{l}\text { Poulet (45 jours) } \\
\text { mâle }\end{array}$ & 155 & 27 \\
femelle & 190 & 35 \\
Dindonneau & & \\
mâle (112 jours) & 70 & 10 \\
femelle (98 jours) & 155 & 22 \\
Pintadeau (84 jours) & 136 & 19 \\
mâle & 200 & 32 \\
femelle & & 35 \\
Caneton de Barbarie & 180 & 42 \\
mâle (84 jours) & 220 & 19 \\
femelle (70 jours) & & 27 \\
Caneton commun (56 jours) & 310 & \\
mâle & 420 & \\
femelle & & \\
\hline
\end{tabular}

dépôts sous-cutanés très développés. Le poulet est en moyenne moins gras et les dépôts souscutanés sont nettement moins importants. Le dindonneau possède des tissus adipeux de taille limitée. Dans tous les cas les femelles sont toujours plus grasses que les mâles. Chez une espèce comme la dinde ce sont elles qui posent le plus de problèmes. Toutes les espèces aviaires présentent un dépôt appelé «gras abdominal » qui tapisse la cavité abdominale depuis le cloaque jusqu'autour du gésier et de l'œsophage. Ce tissu (ou ces tissus) a fait l'objet d'études et de mesures particulières pour plusieurs raisons : 1) c'est un ensemble de tissus qui ne présentent aucun intérêt, contrairement aux tissus sous-cutanés nécessaires à la présentation des carcasses et éventuellement à l'élaboration des arômes du poulet lors de la cuisson, 2) c'est un excellent indicateur de l'état général d'engraissement comme cela a pu être démontré non seulement chez le poulet (Delpech et Ricard 1965, Leclercq 1983) mais aussi chez le canard de Barbarie (Leclercq, non publié). Dans le tableau 1 nous avons rassemblé les valeurs actuelles (mesures récentes) de teneurs en lipides totaux et de proportions de gras abdominal observés chez les différentes espèces exploitées par l'aviculture intensive.

Le gras abdominal est un tissu adipeux à développement tardif comme la plupart des tissus adipeux internes (figure 1). Le gras abdominal, exprimé par rapport au poids vif, est moins développé chez le mâle que chez la femelle. En outre, comme cela a été montré à de multiples occasions, ce développement se ralentit chez le mâle à l'approche de la maturité sexuelle, alors que chez la femelle ce tissu poursuit sa croissance et peut représenter jusqu'à $13 \%$ du poids vif chez des reproductrices lourdes non rationnées. Les triglycérides de réserves contenus dans ce dépôt représentent donc une part croissante des réserves totales du poulet à mesure que l'animal vieillit; cette proportion étant toujours un peu plus élevée chez la femelle (figure 2).
Figure 1. Evolution de la proportion de gras abdominal du poulet de chair en fonction de l'âge (d'après Deaton et Lott 1985).

$$
\begin{aligned}
& \text { gras abdominal } \\
& \text { (en } \% \text { du poids vif) }
\end{aligned}
$$

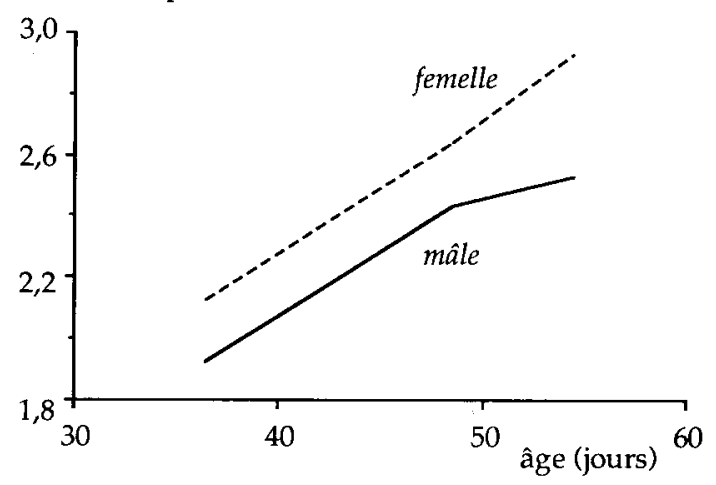

Figure 2. Proportion des lipides de réserve dans le gras abdominal chez le poulet (d'après Leclercq et al 1989).

\section{lipides gras abdominal \\ (en \% lipides totaux)}

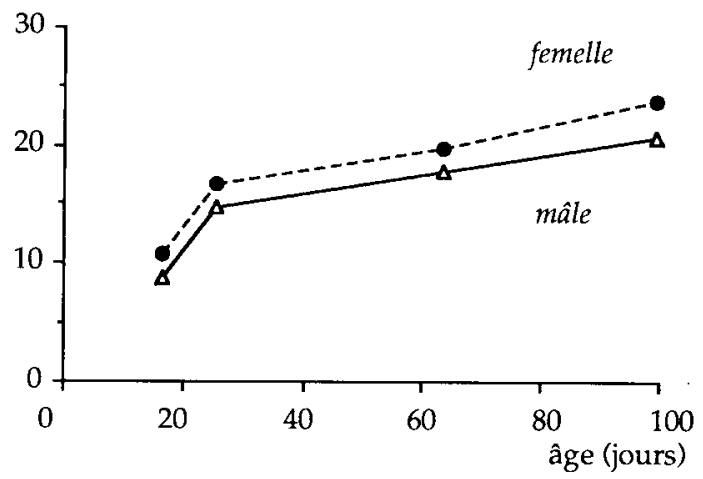


Tableau 2. Evolution des performances de croissance et de l'engraissement des poulets de chair depuis une vingtaine d'années.

\begin{tabular}{|c|c|c|c|c|c|c|}
\hline \multicolumn{2}{|l|}{ Référence } & $\underset{\text { (jours) }}{\text { Age }}$ & $\begin{array}{c}\text { Sexe } \\
(\mathrm{M} \text { ou F })\end{array}$ & $\begin{array}{l}\text { Poids vif } \\
\text { (g) }\end{array}$ & $\begin{array}{c}\text { Gras } \\
\text { abdominal } \\
(\% \\
\text { du poids vif })\end{array}$ & $\begin{array}{c}\text { Lipides } \\
\text { totaux } \\
(\% \\
\text { du poids vif })\end{array}$ \\
\hline Delpech et al & 1965 & 84 & M & 1865 & 0,40 & 7,23 \\
\hline $\begin{array}{l}\text { Ricard et } \\
\text { Rouvier }\end{array}$ & 1969 & $\begin{array}{l}59 \\
59\end{array}$ & $\stackrel{\mathrm{M}}{\mathrm{F}}$ & $\begin{array}{ll}1 & 591 \\
1 & 321\end{array}$ & $\begin{array}{l}0,82 \\
1,10\end{array}$ & - \\
\hline Ricard & 1970 & $\begin{array}{l}77 \\
77\end{array}$ & $\underset{F}{M}$ & $\begin{array}{l}2221 \\
1766\end{array}$ & $\begin{array}{l}1,50 \\
1,90\end{array}$ & - \\
\hline Edwards et al & 1973 & $\begin{array}{l}84 \\
84\end{array}$ & $\stackrel{\mathrm{M}}{\mathrm{F}}$ & $\begin{array}{l}2306 \\
1981\end{array}$ & - & $\begin{array}{r}7,50 \\
13,90\end{array}$ \\
\hline Kubena et al & 1974 & $\begin{array}{l}63 \\
63\end{array}$ & $\stackrel{\mathrm{M}}{\mathrm{F}}$ & $\begin{array}{l}1886 \\
1563\end{array}$ & $\begin{array}{l}1,85 \\
2,36\end{array}$ & - \\
\hline Simon et al & 1978 & $\begin{array}{l}56 \\
56\end{array}$ & $\begin{array}{c}\mathrm{M} \\
\mathrm{F}\end{array}$ & $\begin{array}{l}2268 \\
1793\end{array}$ & $\begin{array}{l}2,40 \\
3,07\end{array}$ & $\begin{array}{l}16,52 \\
18,90\end{array}$ \\
\hline Becker et al & 1979 & $\begin{array}{l}59 \\
59\end{array}$ & $\underset{F}{M}$ & $\begin{array}{l}2287 \\
1920\end{array}$ & $\begin{array}{l}2,00 \\
2,69\end{array}$ & $\begin{array}{r}9,60 \\
11,80\end{array}$ \\
\hline Leeson et al & 1980 & $\begin{array}{l}49 \\
49\end{array}$ & $\underset{\mathrm{F}}{\mathrm{M}}$ & $\begin{array}{l}2123 \\
1766\end{array}$ & $\begin{array}{l}3,40 \\
3,60\end{array}$ & $\begin{array}{l}- \\
-\end{array}$ \\
\hline Deaton et al & 1985 & $\begin{array}{l}48 \\
48\end{array}$ & $\begin{array}{c}\mathrm{M} \\
\mathrm{F}\end{array}$ & $\begin{array}{l}2026 \\
1756\end{array}$ & $\begin{array}{l}2,37 \\
2,77\end{array}$ & - \\
\hline Leclercq et al & 1987 & 42 & M & 1915 & 2,50 & 14,60 \\
\hline $\begin{array}{l}\text { Leclercq } \\
\text { (non publié) }\end{array}$ & 1988 & $\begin{array}{l}46 \\
46\end{array}$ & $\begin{array}{c}\mathrm{M} \\
\mathrm{F}\end{array}$ & $\begin{array}{l}1946 \\
1690\end{array}$ & $\begin{array}{l}3,25 \\
3,64\end{array}$ & $\begin{array}{l}- \\
-\end{array}$ \\
\hline
\end{tabular}

\section{Evolution de l'adiposité au cours de la sélection}

Les espèces d'intérêt avicole ont loutes fait l'objet d'une sélection intensive sur la vitesse de croissance. Chez le poulet le gain génétique est en moyenne de $40 \mathrm{~g}$ par an depuis plus de 30 ans ; ce qui conduit à réduire chaque année d'environ un jour l'âge d'abattage de façon à produire un poulet de poids constant (environ $2 \mathrm{~kg}$ de poids vif). Le canard de Barbarie, dont l'amélioration génétique est plus récente, est, lui, abattu à l'âge fixe (autour de 12 semaines) et à un poids vif de plus en plus élevé. C'est ainsi qu'entre 1974 et 1989 le poids du mâle est passé de $3,4 \mathrm{~kg}$ à $5 \mathrm{~kg}$. La généralisation de la découpe est à l'origine de cet alourdissement de l'espèce. Pour le pintadeau la situation est intermédiaire; l'âge d'abattage a été réduit d'une semaine ef le poids correspondant augmenté $(1,3 \mathrm{~kg}$ à 12 semaines en $1975,1,5 \mathrm{~kg}$ à 11 semaines en 1989). L'utilisation quasi exclusive du dindonneau en découpe le rapproche du caneton de Barbarie de ce point de vue.

L'alourdissement des espèces à un âge donné est donc un phénomène général. Il s'est accompagné d'un développement excessif de l'adiposité; observation manifeste à un âge donné, mais moins flagrante à un poids donné. Nous avons réuni dans le tableau 2 des références fournissant entre 1965 et 1988 les critères d'adiposité du poulet de chair à l'âge d'abattage dans les conditions normales d'élevage. Les comparaisons dans le temps sont toujours délicates puisque, si l'évolution des génolypes est manifeste, celles des conditions d'élevage n'est pas négligeable non plus. Fn particulier on sait depuis longtemps que la leneur en protéines des alimenls est susceptible de modifier notablement l'adiposité des poulets. Une inflation certaine des apports protéiques au-delà du besoin de croissance peut être observée depuis une vingtaine d'années. Elle correspond essentiellement à la nécessité de réduire l'engraissement et d'améliorer l'efficacité alimentaire. C'est pourquoi parmi les données des références du tableau 2 nous n'avons relenu que

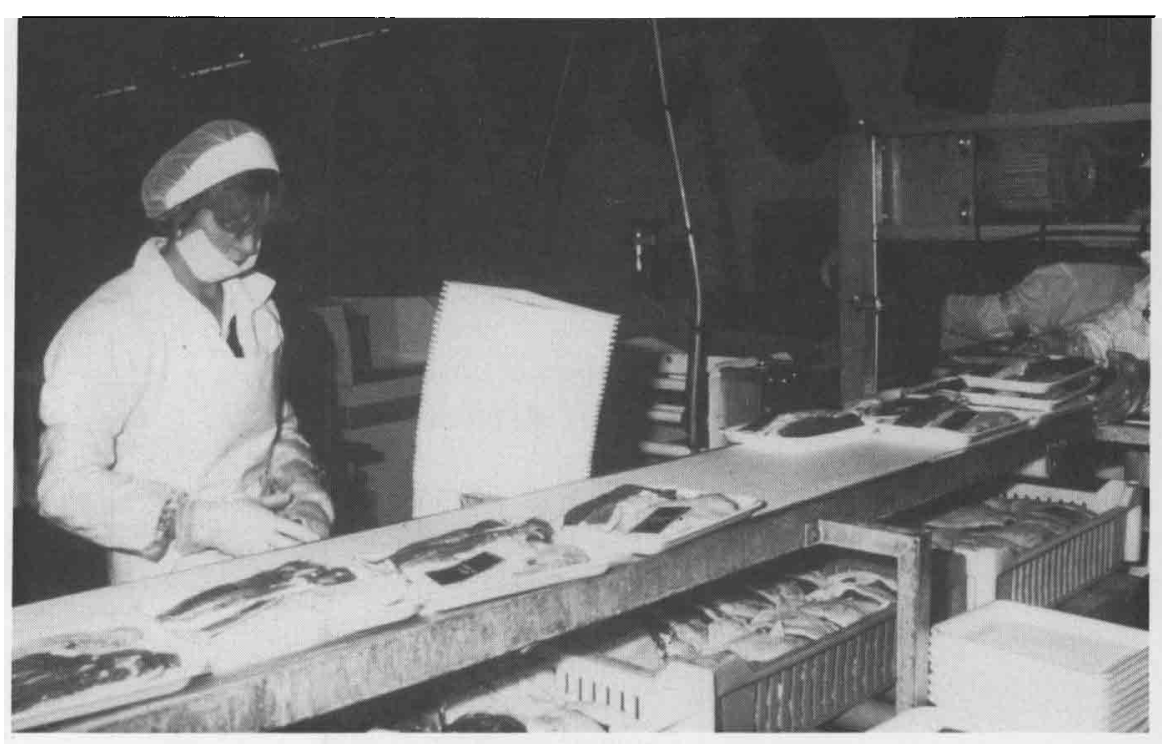

Le canard de Barbarie est abattu à àge fixe, à un poids vit de plus en plus élevé. La généralisation de la découpe est à l'origine de cet alourdissement de l'espèce, qui s'accompagne d'un développement excessif de l'adiposité. 
Tableau 3. Quelques valeurs de la corrélation entre état d'engraissement et vitesse de croissance chez le poulet.

\begin{tabular}{|l|c|c|}
\hline \multicolumn{1}{|c|}{ Référence } & \multicolumn{2}{|c|}{ Paramètre d'engraissement } \\
& $\begin{array}{c}\text { Lipides totaux/ } \\
\text { poids vif }\end{array}$ \\
\hline $\begin{array}{l}\text { Van Middelkoop et al (1976) } \\
\text { poids vif }\end{array}$ & \\
mâles & $0,21^{* *}$ & \\
femelles & $0,18^{* *}$ & $0,32^{\star *}$ \\
Pym et Solvyns (1980) & & \\
Leclercq et al (1980) & & \\
mâles & $0,59^{* *}$ & \\
femelles & $0,69^{* *}$ & \\
Friars et al (1983) & $0,18 \mathrm{~ns}$ & \\
Becker et al (1984) & & \\
mâles & $0,42^{* *}$ & \\
femelles & $0,47^{* *}$ & $0,41 \mathrm{~ns}$ \\
Leenstra et al (1986) & & $0,61^{* *}$ \\
mâles & $0,46^{*}$ & \\
femelles & $0,44 \mathrm{~ns}$ & \\
\hline
\end{tabular}

${ }^{*}$ : significatif au seuil de $5 \% \quad * *$ : significatif au seuil de $1 \%$. ns : non significatif.

L'état

d'engraissement est positivement corrélé au poids vif à un âge donné. La sélection sur la vitesse de croissance a donc conduit à sélectionner les animaux les plus gras.
Tableau 4. Corrélations entre le poids vif à 12 semaines et quelques caractéristiques de carcasse du canard de Barbarie. (d'après Leclercq et de Carville 1978).

\begin{tabular}{|l|c|}
\hline & Poids vif \\
& à 12 semaines \\
\hline Gras abdominal/poids vif & $0,64^{* *}$ \\
Filets avec peau/poids vif & $0,20^{\star}$ \\
Pilons + cuisses/poids vif & $-0,20^{\star}$ \\
\hline
\end{tabular}

${ }^{*}$ : significatif au seuil de $5 \%$.

** : significatif au seuil de $1 \%$.

Tableau 5. Estimations de l'héritabilité (calculée) de l'engraissement chez le poulet de chair.

Paramètres : GA/P : gras abdominal divisé par poids vif ; D (GA/P) : écart à la régression linéaire entre gras abdominal et poids vif ; LT /P : lipides totaux divisés par poids vif; D (GA.P/P) : écart à la régression linéaire entre gras abdominal divisé par poids vif et poids vif.

\begin{tabular}{|l|c|c|c|}
\hline \multicolumn{1}{|c|}{ Référence } & Paramètre & Sexe & $\mathbf{h}^{2}$ combinée \\
\hline Ricard et Rouvier (1967) & $\mathrm{D}(\mathrm{GA} / \mathrm{P})$ & $\mathrm{M}$ & 0,47 \\
Ricard et Rouvier (1969) & $\mathrm{D}(\mathrm{GA} / \mathrm{P})$ & $\mathrm{M}$ & 0,72 \\
Leclercq et al (1980) & & $\mathrm{F}$ & 0,71 \\
& $\mathrm{GA} / \mathrm{P}$ & $\mathrm{M}$ & 0,98 \\
Chambers et Gavora (1984) & $\mathrm{D}(\mathrm{GA} . \mathrm{P} / \mathrm{P})$ & $\mathrm{M}+\mathrm{F}$ & 0,71 \\
Friars et al (1983) & $\mathrm{GA} / \mathrm{P}$ & $\mathrm{F}$ & 0,64 \\
& $\mathrm{GA} / \mathrm{P}$ & $\mathrm{F}$ & 0,62 \\
Leclercq et al (1983) & $\mathrm{LT} / \mathrm{P}$ & $\mathrm{M}$ & 0,48 \\
(non publié) & $\mathrm{GA} / \mathrm{P}$ & $\mathrm{F}$ & 0,44 \\
Becker et al (1984) & $\mathrm{GA} / \mathrm{P}$ & $\mathrm{F}$ & 0,63 \\
& & $\mathrm{M}$ & 0,79 \\
Gyles et al (1984) & $\mathrm{LT} / \mathrm{P}$ & $\mathrm{F}$ & 0,78 \\
Cahaner et Nitsan (1985) & $\mathrm{GA} / \mathrm{P}$ & $\mathrm{M}(\mathrm{White} \mathrm{Rock})$ & 0,72 \\
Leenstra et al (1986) & $\mathrm{M}$ (Cornish) & 0,23 \\
\hline
\end{tabular}


les valeurs correspondant aux aliments de caractéristiques proches des conditions usuelles d'élevage. Ce tableau montre nettement une réduction régulière de l'âge d'abattage, alors que les poids vifs demeurent pratiquement constants. Parallèlement on observe une augmentation, certes moins régulière mais significative, de la proportion du gras abdominal et dos lipides totaux.

Il existe plusieurs explications de cet accroissement régulier de l'adiposité. La première est la corrélation positive entre état d'engraissement et poids vif à un âge donné. Quelques références sont fournies à ce sujet pour le poulet dans le tableau 3. Si quelques auteurs ne trouvent pas de corrélation significative, la plupart observent des valeurs positives proches de $+0,5$. Il s'ensuit que le fait de retenir lors de la sélection les animaux les plus lourds comme parents de la génération suivante entraîne en même temps la sélection des géniteurs les plus gras. Des observations identiques ont été faites chez le canard de Barbarie mâle, chez lequel une corrélation hautement significative de + 0,64 a été calculée entre le poids vif et la proportion de gras abdominal (Leclercq et de Carville 1978), comme cela est rapporté dans le tableau 4. Il en est de même aussi chez le pintadeau chez qui les corrélations phénotypiques se situent entre $+0,5$ et $+0,7$ à l'âge de 12 semaines (Blum et Leclercq 1978).

Une seconde explication possible, qui n'exclut pas la précédente, réside dans la modification de la forme de la courbe de croissance. En effet, chez le poulet, la sélection sur la vitesse de croissance s'accompagnant d'un raccourcissement de la durée d'élevage tend à accélérer la vitesse de croissance au cours de la phase précoce de cette courbe. Or, il semble exister une corrélation entre la forme de la courbe de croissance et l'adiposité. Comme l'a montré Ricard (1978) en sélectionnant, à partir d'une même population de base, des lignées à courbes de croissance de formes différentes, les poulets à croissance initiale rapide et poids vif mature faible sont nettement plus gras que les poulets à croissance initiale lente et à poids vif mature élevé. L'observation réciproque a été réalisée par nous-même sur deux lignées sélectionnées de façon divergente sur la proportion de gras abdominal à l'âge de 9 semaines : la lignée maigre présente une croissance initiale plus lente que la lignée grasse, mais parvient à un poids adulte plus élevé (Leclercq et al 1989).

\section{Héritabilité de l'état d'engraissement et sélections expérimentales}

Les équipes de recherches françaises se sont les premières préoccupé du contrôle génétique de l'engraissement. Dès 1967, Ricard et Rouvier (1967 et 1969) ont estimé l'héritabilité de l'ensemble des caractéristiques anatomiques chez deux lignées de poulets : le Bresse-Pile (1967) et le Cornish (1969). Parmi les nombreux paramètres étudiés figure le « gras abdominal » qui, à l'époque, présentait un développement très modéré. Le critère retenu a été la déviation par rapport à la droite de régression linéaire entre le « gras abdominal » et le poids vif. L'héritabilité de ce critère a été trouvée égale ou supérieure à 0,50 . Les valeurs exactes sont rapportées dans le tableau 5 ; nous y avons joint les observations réalisées à partir de 1980 par plusieurs équipes à partir du moment où le développement du tissu adipeux a posé des problèmes sérieux à la production avicole. Les critères utilisés sont soit le rapport gras abdominal/poids vif, soit lipides totaux/poids vif, soit l'écart à la régression linéaire entre gras abdominal et poids vif soit enfin l'écart à la régression entre le rapport gras abdominal/poids vif et le poids vif. Tous ces critères présentent des valeurs calculées de l'héritabilité très élevées et

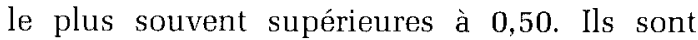
confirmés рат les valeurs des héritabilités réalisées (Leclercq 1988, Cahaner 1988). Il est donc manifeste qu'une sélection contre l'adiposité est tout à fait envisageable. Il convient cependant: 1) de trouver une méthode aisée d'évaluation des dépôts adipeux que l'on souhaite réduire, 2) d'estimer les réponses d'autres paramètres zootechniques, en particulier des réponses défavorables, qui sont corrélés avec les dépôts gras.

\section{Méthodes de sélection contre l'adiposité}

Le principal problème rencontré pour la mise en ceuvre pratique d'une sélection contre l'adiposité est une estimation de l'état d'engraissement qui soit rapide et non destructive, c'est-àdire qui puisse être appliquée à l'animal vivant, candidat à la reproduction. Il faut, de plus, qu'elle puisse être mise en œuvre sur de grands effectifs (plusieurs milliers). A l'heure actuelle ce problème n'a pas encore trouvé de solution satisfaisante chez le poulet.

La seule méthode envisageable aujourd'hui est basée sur l'indice de consommation (rapport entre consommation d'aliment et gain de poids]. En effet, le coût énergétique d'un gramme de tissu non adipeux est nettement

Dans la sélection expérimentale réalisée en France, 2 lignées ont été obtenues par sélection divergente: l'une en vue d'accroître le gras abdominal, l'autre en vue de le réduire. Au bout de 7 générations, le gras abdominal était dans un rapport de 1 à 4 entre génotype maigre et génotype gras.

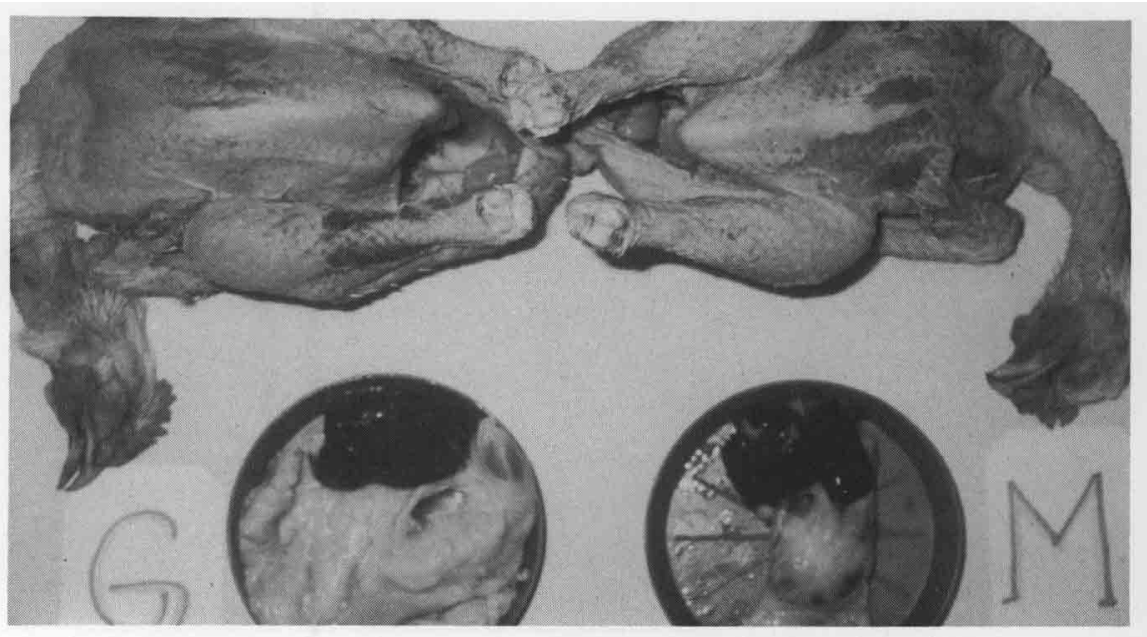


inférieur à celui d'un gramme de tissu adipeux (en moyenne 2,8 contre $10 \mathrm{kcal} / \mathrm{g}$ ). Ceci est dû surtout au fait que les tissus non adipeux renferment principalement de l'eau (65\%) et des protéines $(18 \%)$; alors que les tissus adipeux renferment surtout des lipides $(85 \%$ en moyenne) et peu d'eau (environ $10 \%$ ). Comme le coût énergétique de synthèse d' $1 \mathrm{~g}$ de lipides et de $1 \mathrm{~g}$ de protéines sont assez proches, compte tenu des rendements énergétiques très différents, le dépôt d'1 g de tissu adipeux est 3 à 4 fois plus coûteux du point de vue énergétique que celui de $1 \mathrm{~g}$ de tissu non adipeux. On doit donc s'attendre à ce que des génotypes maigres présentent, toutes choses étant égales par ailleurs (en particulier la vitesse de croissance), un indice de consommation inférieur à celui des génotypes gras. C'est ce que confirment toutes les observations faites sur lignées expérimentales. Réciproquement les sélections expérimentales réalisées sur l'indice de consommation aboutissent à la production d'animaux qui tendent à être maigres.

L'efficacité alimentaire (ou l'indice de consommation qui est son inverse) est un caractère à héritabilité moyenne (entre 0,30 et 0,40 ). Nous avons rassemblé dans le tableau 6 les principales références sur ce sujet, qu'il s'agisse d'héritabilité calculée ou réalisée. Selon les auteurs les valeurs vont de 0,20 à 0,70 . La principale raison de ces différences doit venir vraisemblablement des dispositifs expérimentaux utilisés. En effet dans ce type d'expérience on doit disposer de plusieurs centaines (voire milliers) de cages individuelles. Or dans de telles installations on observe très souvent des effets du milieu (effet de façade, d'étage...) plus ou moins importants qui risquent de réduire l'héritabilité s'ils sont ignorés dans les calculs ou s'ils ne sont pas réduits par une conception judicieuse du dispositif.

La sélection sur l'indice de consommation est donc possible. Deux sélections expérimentales ont été réalisées récemment, l'une en Australie (Pym et al 1979), l'autre en Hollande (Leenstra 1988). Cette méthode de sélection présente de nombreux avantages et quelques inconvénients. Elle permet de réduire l'état d'engraissement et le besoin énergétique d'entretien des poulets. Elle augmente la digestibilité des lipides alimentaires (Leenstra et al 1988). De plus, comme l'indice de consommation est corrélé avec la vitesse de croissance (corrélation phénotypique de - 0,25), la sélection vers une réduction de cet indice tend aussi à augmenter la vitesse de croissance. Enfin ce type de sélection réduit significativement la fréquence et la gravité de la dyschondroplasie, l'un des troubles locomoteurs les plus répandus chez le poulet (Leenstra 1988). Ce phénomène n'a pas trouvé d'explication. Un des inconvénients de la sélection sur l'indice de consommation est qu'elle réduit indistinctement tous les tissus adipeux, alors qu'un effort spécifique doit être fait contre le tissu abdominal. En revanche la présence d'un minimum de tissus adipeux sous-cutanés est souvent souhaitée chez la plupart des espèces avicoles.

D'autres méthodologies de sélection sont envisageables pour réduire l'engraissement. L'une d'entre elles, basée sur la mesure des lipoprotéines de très basse densité (VLDL) a été tentée par Whitehead et al (1982) chez le poulet. Chez des poulets nourris, la teneur du plasma en VLDL présente une corrélation de l'ordre de $+0,6$ avec l'engraissement. L'héritabilité de ce constituant plasmatique est en moyenne de 0,50 (Whitehead 1988). Il s'agit donc d'une mesure indirecte de l'engraissement qui a l'avantage de ne pas perturber l'animal. Plusieurs inconvénients doivent toutefois être signalés. Tout d'abord ce critère de sélection semble plus efficace pour sélectionner des animaux gras que des animaux maigres (Whitehead et Griffin 1988). Comme la sélection sur l'indice de consommation, celle qui est basée sur les VLDL tend à réduire indistinctement tous les tissus adipeux, sans effet spécifique sur le gras abdominal, dépôt à supprimer en priorité. En outre cette méthode oblige à prélever un échantillon de sang dans des conditions très précises d'alimentation. Enfin, même correctement automatisé, le dosage prend un certain temps. D'autres paramètres plasmatiques ont

Tableau 6. Héritabilité calculée $(C)$ ou réalisée $(R)$ de l'efficacité alimentaire (gain de poids/consommation d'aliment) ou de l'indice de consommation (consommation/gain de poids) chez le poulet.

\begin{tabular}{|l|c|c|}
\hline \multicolumn{1}{|c|}{ Référence } & Période de mesure & $\mathbf{h}^{2}$ combinée \\
\hline Thomas et al (1958) & $4-6$ semaines & $0,42 \mathrm{C}$ \\
& $6-8$ semaines & $0,25 \mathrm{C}$ \\
& $8-10$ semaines & $0,23 \mathrm{C}$ \\
Wilson (1969) & $4-8$ semaines & $0,43 \mathrm{C}$ \\
Guill et Washburn (1974) & $6-10$ semaines & $0,40 \mathrm{C}$ \\
& $5-10$ semaines & $0,42 \mathrm{C}$ \\
& $4-8$ semaines & $0,20 \mathrm{R}$ \\
Pym et Nicholls (1979) & $4-8$ semaines & $0,21 \mathrm{R}$ \\
Chambers et Gavora (1982) & $4-8$ semaines & $0,33 \mathrm{R}$ \\
Leclercq et al (non publié) & $4-8$ semaines & $0,26 \mathrm{R}$ \\
Leenstra et al (1986) & $5-9$ semaines & $0,21 \mathrm{R}$ \\
& $4-7$ semaines & $0,37 \mathrm{C}$ \\
& $3-7$ semaines & $0,70 \mathrm{C}$ \\
\hline
\end{tabular}


été proposés. Les triglycérides plasmatiques (Leclercq et Guy 1988) sont corrélés avec la proportion de gras abdominal (en moyenne $\mathrm{r}=$ $+0,50$ ). Les phospholipides plasmatiques, témoins de la concentration en lipoprotéines de haute densité (HDL), pourraient eux aussi, être utilisés (Leclercq et Guy 1988). Ils ne sont pas influencés par l'état nutritionnel (à jeun ou nourri) et présentent une corrélation positive avec l'état d'engraissement $(+0,30)$. Enfin chez le poulet la sélection sur la glycémie basale peut modifier l'état d'engraissement: les animaux à glycémie élevée étant maigres (Leclercq et al 1987). Quels qu'ils soient, les paramètres plasmatiques présentent des limites d'interprétation et d'utilisation. La concentration d'un constituant du plasma est la résultante d'entrées et de sorties du pool plasmatique. La sélection risque donc de modifier les unes et les autres en même temps, rendant l'efficacité de ces méthodes incertaine à moyen terme. Elles peuvent cependant rendre des services à court terme; par exemple l'élimination des poulets à concentrations plasmatiques élevées de VLDL doit certainement contribuer à supprimer des animaux très gras et abaisser ainsi l'état moyen d'adiposité de la population.

La sélection sur collatéraux a fait ses preuves en sélection expérimentale. Quatre équipes de recherches l'ont pratiquée (Cahaner 1988 , Leclercq 1988, Leenstra 1988, Lilburn 1988). Théoriquement cette méthode, qui consiste à estimer le génotype d'un candidat à la reproduction d'après la valeur de $\mathrm{n}$ frères ou demifrères sacrifiés pour la circonstance, représente une perte d'efficacité et un coût non négligeable (Mallard et Douaire 1988). Son intérêt est néanmoins à prendre en considération du fait : 1) de l'héritabilité très élevée du rapport gras abdominal/poids vif, 2) de l'efficacité observée lors des sélections expérimentales, 3) de la réduction plus spécifique du dépôt abdominal par rapport aux autres tissus. La mesure associée d'autres critères de carcasse, tels que le développement des muscles pectoraux peut redonner de l'intérêt aux mesures sur collatéraux en attendant la découverte de méthodes non destructives. Enfin il faut rappeler qu'il existe toujours une corrélation positive et modérée entre poids vif et état d'adiposité, tout au moins chez les espèces à faible adiposité comme les volailles, qui fait qu'une sélection vers la réduction de l'engraissement tend à se faire un peu au détriment de la vitesse de croissance. Heureusement la valeur de la corrélation est suffisamment faible pour permettre une sélection spécifique de l'engraissement. Le tableau 7 résume les avantages respectifs de la sélection sur l'indice de consommation et de la sélection sur le dépôt gras abdominal.

En conclusion il est probable que la solution pratique soit peut-être la combinaison de plusieurs méthodes de sélection. Selon l'espèce et selon la stratégie du sélectionneur plusieurs combinaisons dans le temps ou à un instant donné peuvent être en effet envisagées.

\section{Intérêts zootechniques et technologiques des génotypes maigres}

\section{Efficacité alimentaire}

Le premier intérêt zootechnique des génotypes maigres est leur meilleure efficacité alimentaire. Quel que soit le mode de sélection utilisé l'amélioration est substantielle et dépend, bien évidemment de l'intensité de la sélection vers la maigreur. Dans la sélection expérimentale réalisée en France, deux lignées ont été obtenues par sélection divergente, l'une en vue d'accroître le gras abdominal, l'autre en vue de la réduire. Partant d'une population ou le rapport gras abdominal/poids vif était de 2,7\% chez les mâles âgés de 62 jours il a été possible en 7 générations de parvenir à une lignée maigre où il est de $1 \%$. S'il existe un rapport de 1 à 4 pour le gras abdominal entre génotype maigre et génotype gras, le rapport entre lipides totaux n'est que de 1 à 2 . Dans ces conditions la différence d'indice de consomma-

Tableau 7. Avantages respectifs d'une sélection soit sur l'indice de consommation, soit sur la proportion de graisse abdominale.

\begin{tabular}{|c|c|c|}
\hline & $\begin{array}{l}\text { Réduction de l'indice } \\
\text { de consommation }\end{array}$ & $\begin{array}{c}\text { Réduction } \\
\text { de la graisse abdominale }\end{array}$ \\
\hline Poids vif & corrélation positive & corrélation légèrement négative \\
\hline Digestibilité des graisses & améliorée $[+3 \%)$ & inchangée \\
\hline Besoin énergétique d'entretien & réduit & inchangé \\
\hline Indice de consommation & très amélioré $(-0,150)$ & amélioré $(-0,100)$ \\
\hline Adiposité : & & \\
\hline $\begin{array}{l}\text { - générale } \\
\text { - gras abdominal }\end{array}$ & $\begin{array}{l}\text { réduile } \\
\text { réduile }\end{array}$ & $\begin{array}{l}\text { réduite } \\
\text { spécifiquement réduite }\end{array}$ \\
\hline Mortalité & inchangée & inchangée \\
\hline Troubles locomoteurs & réduits & légèrement réduits \\
\hline Rendement en carcasse & amélioré (+ $10 \mathrm{~g} / \mathrm{kg})$ & amélioré (+ 10 g/kg) \\
\hline Rendement en filets & amélioré (+ $10 \mathrm{~g} / \mathrm{kg}$ ) & amélioré $(+10 \mathrm{~g} / \mathrm{kg})$ \\
\hline
\end{tabular}


La sélection sur l'indice de consommation réduit également la fréquence et la gravité de la dyschondroplasie, lésion des pattes qui entraîne fréquemment des fractures lorsque les animaux sont accrochés sur les chaines à l'abattoir.

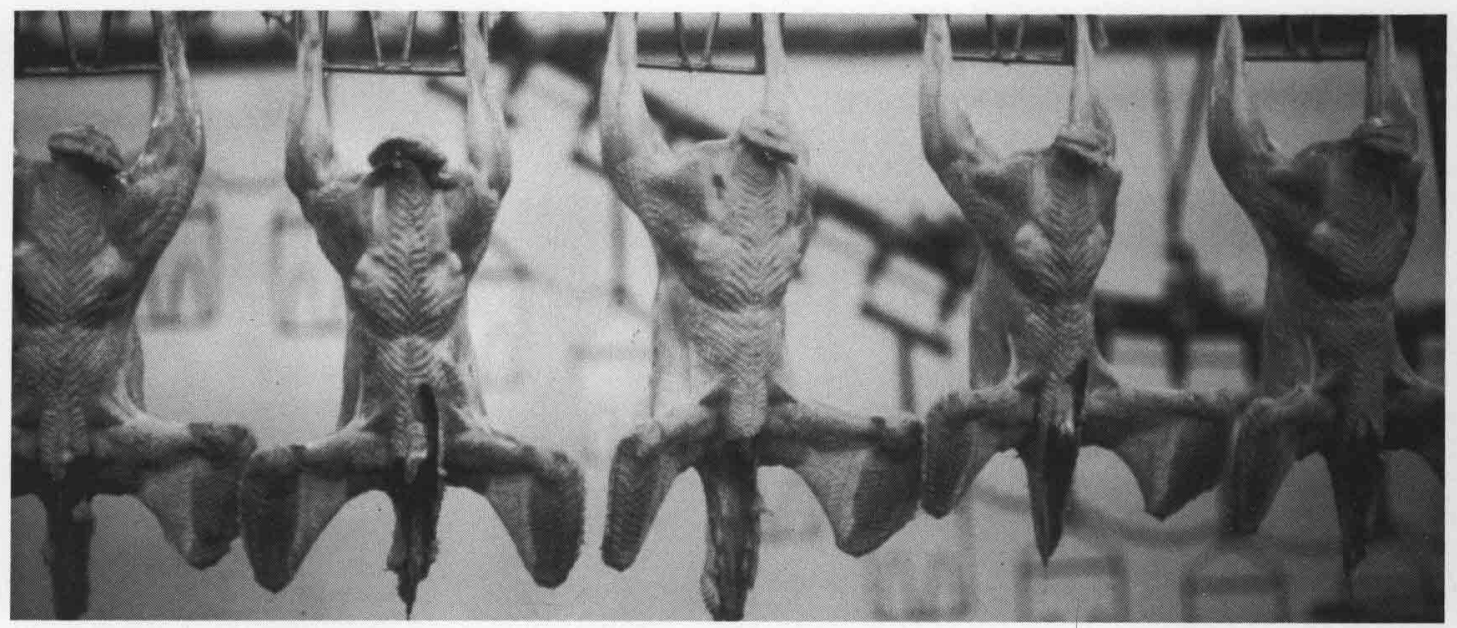

tion est en moyenne de 0,20 (200 g d'aliment par $\mathrm{kg}$ de poids vif). Si on admet que la sélection a été à peu près symétrique, le gain par rapport à la lignée de départ est de moitié, soit 0,10 , c'est-à-dire une économie de $100 \mathrm{~g}$ d'aliment par $\mathrm{kg}$ de poulet produit. Dans l'expérience de sélection réalisée en Ecosse et basée sur les VLDL plasmatiques les lipides corporels de la lignée maigre représentent $13,4 \%$ du

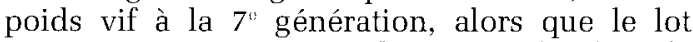
témoin est à $16,4 \%$. La différence d'indice de consommation est de 0,12 (Whitehead 1988). Dans l'expérience hollandaise (Leenstra 1988) la lignée sélectionnée pour un faible pourcentage de gras abdominal présente un rapport de 1 à 2,2 pour ce critère par rapport au lot témoin; la différence d'indice de consommation est de 0,11 mais elle doit être un peu moins prononcée si on raisonne à poids vif égal, la lignée témoin étant nettement plus lourde que la lignée maigre. Au cours de cette même expérience la lignée sélectionnée pour une efficacité alimentaire élevée se caractérise par un rapport de 1 à 1,55 pour la proportion de gras abdominal; la différence d'indice est alors de 0,17 par rapport à la lignée témoin. Cette différence doit probablement être un peu plus faible si on raisonne à poids vif équivalent pour la même raison que précédemment. On peut donc conclure qu'une sélection menée de façon intensive contre l'engraissement peut permettre d'économiser au moins $100 \mathrm{~g}$ d'aliment par $\mathrm{kg}$ de poulet. Le gain doit certainement être supérieur si l'on associe la sélection sur la maigreur et la sélection sur l'efficacité alimentaire.

\section{Economie de protéines alimentaires}

L'une des retombées inattendues de ces sélections expérimentales concerne l'utilisation des protéines alimentaires par les lignées maigres. Dans le tableau 8 nous avons rassemblé les valeurs du rapport protéines fixées/protéines ingérées observées au cours de 5 expériences de sélection réalisées sur poulet de chair. Une seule expérience comporte un lot témoin vis-à-vis de lignée maigre. Les autres associent une lignée maigre à une lignée grasse ; ce qui rend un peu plus difficile l'interprétation des phénomènes, car on ne sait pas avec certitude si la divergence vient de la lignée maigre, de la lignée grasse ou des deux lignées à la fois.

Quoi qu'il en soit, il ressort nettement de ce tableau, et plus encore des publications citées, que les lignées maigres transforment les protéines alimentaires en protéines corporelles avec une efficacité nettement supérieure aux lignées grasses ou aux lignées témoins non sélectionnées. Ce phénomène est confirmé par Whitehead (1988) chez les lignées sélectionnées sur les VLDL plasmatiques : l'efficacité est de 0,34 chez la lignée grasse et de 0,40 chez la lignée maigre. En moyenne on peut considérer que, par rapport à une lignée témoin, le rendement de transformation des protéines alimentaires en protéines de carcasse est amélioré de $3 \%$; ce qui peut se traduire par une économie de $25 \mathrm{~g}$ de protéines alimentaires par $\mathrm{kg}$ de poulet. Il s'ensuit que les poulets maigres

Tableau 8. Valeurs du rapport protéines fixées sur protéines ingérées chez plusieurs lignées expérimentales de poulets différant pour leur état d'engraissement.

\begin{tabular}{|l|c|c|c|}
\hline \multicolumn{1}{|c|}{ Référence } & grasse & $\begin{array}{c}\text { Lignée } \\
\text { témoin }\end{array}$ & maigre \\
\hline Leclercq $(1983)^{*}$ & 0,38 & & 0,41 \\
Pym et al $(1984)^{* *}$ & & 0,53 & 0,56 \\
Mc Leod et al $(1988)^{*}$ & 0,31 & & 0,40 \\
Geraert et al $(1988$ a) & 0,39 & & 0,45 \\
Sorensen et al $(1983)^{* *}$ & 0,54 & 0,60 & \\
\hline
\end{tabular}

\footnotetext{
* protéines corporelles à l'exclusion des plumes.
}

** calcul basé sur les protéines digestibles. 
Figure 3. Influence de la teneur en protéines brutes de l'aliment sur lindice de consommation (IC) de poulets génétiquement gras ou maigres.
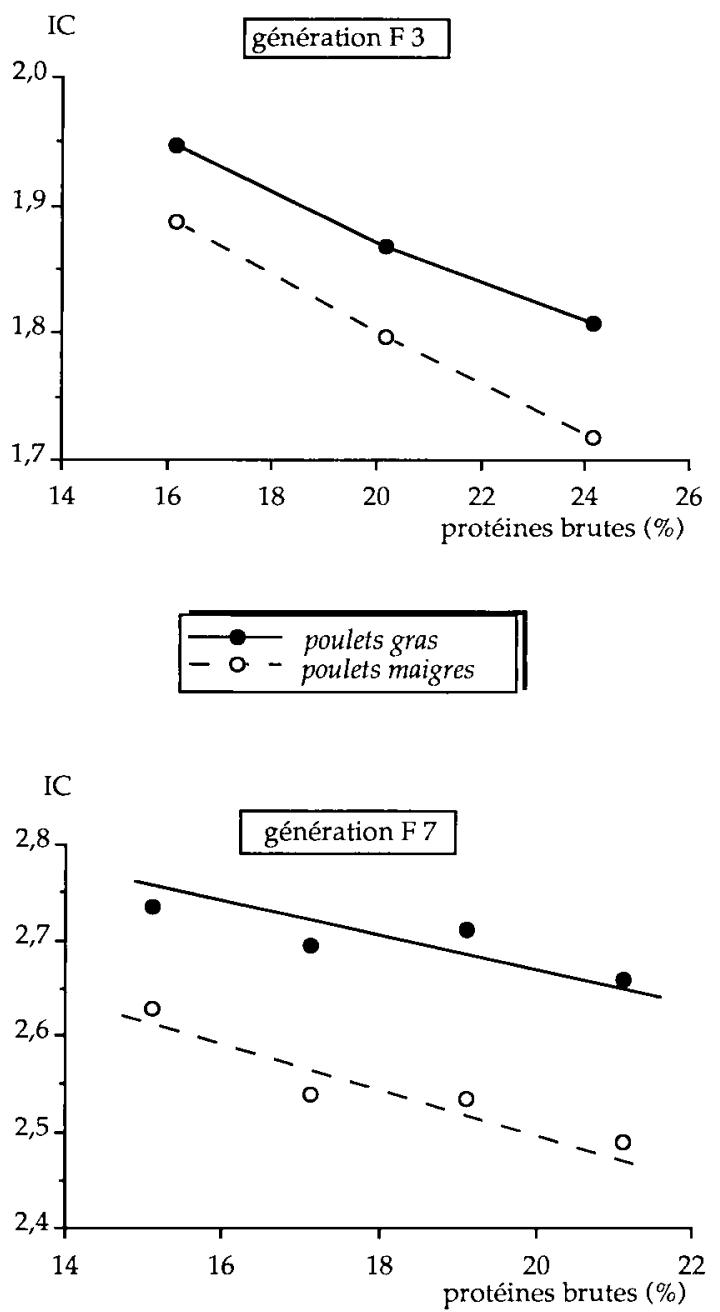

excrètent moins d'acide urique (Geraert et al 1988 b). Cette caractéristique peut s'avérer intéressante pour lutter contre la pollution azotée par les fientes dans les zones d'élevage intensif.

Les mécanismes qui sont à l'origine de cette utilisation plus efficace des acides aminés alimentaires à des fins de protéinogénèse font l'objet actuellement d'investigations. Il a été montré que les lignées grasses incorporent significativement plus de carbone 14 des acides aminés alimentaires dans leurs acides gras de réserve (Mammier et al 1985, Geraert et al 1986). Il y a donc chez eux une déviation métabolique, probablement sous contrôle hormonal, qui dirige systématiquement une part des acides aminés vers la production d'acétylcoenzyme A et la lipogenèse.

Si les lignées maigres sont de meilleurs transformateurs des acides aminés alimentaires en protéines corporelles, elles sont en revanche plus sensibles à la teneur de l'aliment en protéines brutes dans les zones de variation endessous du besoin usuel (Leclercq 1983, Whitehead 1988). La réciproque avait été observée dès 1980 par Sorensen qui, voulant sélectionner des poulets économes en protéines, avait effectué une sélection en régime carencé en protéines. Après 3 générations il observa qu'il avait en fait sélectionné une lignée grasse et moins efficace dans l'utilisation des protéines alimentaires. Les phénomènes décrits précédemment constituent une bonne illustration des interactions possibles entre le milieu et la sélection.

Apparemment les lignées maigres et grasses réagissent de façon parallèle aux changements du taux protéique alimentaire pour ce qui concerne l'indice de consommation et l'état d'engraissement. A cette occasion on peut aisément comprendre l'intérêt pratique d'une sélection contre l'adiposité. La figure 3 décrit l'évolution de l'indice de consommation lorsque les teneurs en protéines brutes de l'aliment sont modifiées; les données proviennent de 2 expériences réalisées sur nos lignées en génération F3 et F7. On remarque l'évolution parallèle des lignées, l'écart étant plus prononcé en F7 qu'en F3. En F3 il faut en moyenne relever de $40 \mathrm{~g} / \mathrm{kg}$ la teneur en protéines de l'aliment distribué à des animaux génétiquement gras pour réduire l'indice de consommation au niveau des animaux maigre. En F7 c'est une élévation de $100 \mathrm{~g} / \mathrm{kg}$ qui devient nécessaire. Ces chiffres doivent être divisés par 2 pour obtenir un ordre de grandeur d'une comparaison avec une lignée témoin. Cette figure illustre donc l'intérêt respectif des deux voies envisagées pour réduire l'indice de consommation du poulet. La voie nutritionnelle, basée sur la distribution d'excès de protéines, trouve ici sa limite pratique au bénéfice de la voie génétique, plus longue à mettre en œuvre mais moins gaspilleuse en ressources protéiques.

Un phénomène semblable est illustré par la figure 4, rapportant des observations faites aussi en F3 et en F7. Les différences entre lignées sont encore plus prononcées que pour l'indice de consommation. En F3 il faut augmenter de $55 \mathrm{~g} / \mathrm{kg}$ la teneur en protéines brutes des aliments distribués au génotype gras pour réduire son engraissement au niveau du génotype maigre. En F7 l'augmentation théorique ne peut être estimée puisqu'il n'y a pas recouvre-

Figure 4. Influence de la teneur de l'aliment en protéines brutes sur l'état d'engraissement des lignées de poulets différant génétiquement pour l'état d'adiposité.

gras abdominal (\% du poids vif)

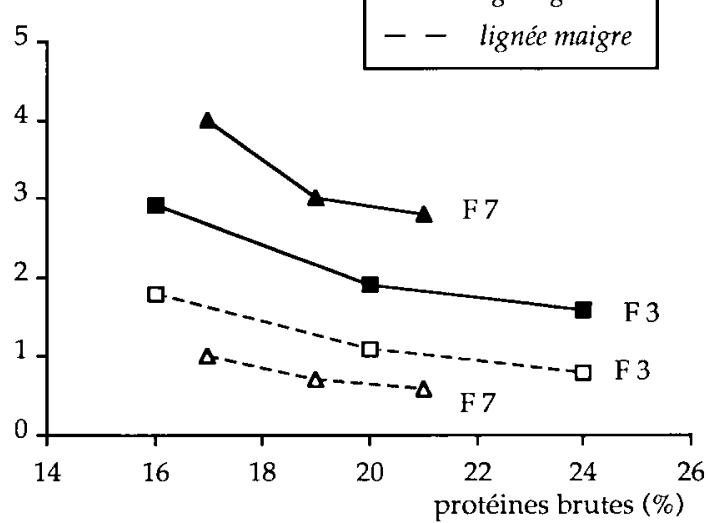


Tableau 9. Résultats de dissection de mâles de 2 lignées expérimentales de poulets différant pour leur état d'engraissement (Ricard et Touraille 1988).

\begin{tabular}{|c|c|c|c|c|c|c|}
\hline & \multicolumn{3}{|c|}{ Génération 5} & \multicolumn{3}{|c|}{ Génération 7} \\
\hline & gras & & maigre & gras & & maigre \\
\hline Poids vif $(g)$ & 1774 & ns & 1862 & 1778 & ns & 1745 \\
\hline Carcasse éviscérée/poids vif & 0,593 & * & 0,602 & 0,612 & ns & 0,620 \\
\hline $\begin{array}{l}\text { Muscles pectoraux/carcasse } \\
\text { éviscérée }\end{array}$ & 0,188 & ** & 0,198 & 0,194 & ** & 0,206 \\
\hline $\begin{array}{l}\text { Cuisses+ pilons/carcasse } \\
\text { éviscérée }\end{array}$ & 0,392 & ns & 0,398 & 0,390 & ns & 0,385 \\
\hline $\begin{array}{l}\text { Muscles cuisses + pilons/ } \\
\text { carcasse éviscérée }\end{array}$ & 0,622 & ** & 0,648 & 0,612 & $* *$ & 0,638 \\
\hline
\end{tabular}

* significatif au seuil de $5 \%, \quad$ ** significatif au seuil de $1 \%$, ns non significatif.

Tableau 10. Rendement en viande de filets des lignées maigres expérimentales (en \% du poids vif).

\begin{tabular}{|l|c|c|c|}
\hline \multicolumn{1}{|c|}{ Référence } & grasse & $\begin{array}{c}\text { Lignée } \\
\text { témoin }\end{array}$ & maigre \\
\hline Cahaner et al (1986) & $14,8 \%$ & & $15,6 \%$ \\
Leenstra et al (1987) & & $13,9 \%$ & $14,2 \%$ \\
Ricard et al (1988) & $11,5 \%$ & & $12,3 \%$ \\
\hline
\end{tabular}

ment des deux courbes de régression. L’intérêt de la solution génétique du problème de l'engraissement devient alors encore plus manifeste que pour l'indice de consommation.

\section{Influence sur les qualités de carcasse}

La sélection de lignées maigres réduit bien évidemment les tissus adipeux. Nous avons évoqué précédemment l'incidence de la méthodologie de sélection sur la répartition des divers tissus adipeux, en particulier entre tissus sous-cutanés et tissus internes (gras abdominal). Toutefois cette sélection ne se limite pas à ces seules conséquences. Elle entraîne aussi une amélioration du rendement en carcasse (poids de la carcasse éviscérée/poids vif), un développement un peu plus important mais significatif des muscles pectoraux et de l'ensemble muscles pectoraux + muscles des membres postérieurs (cuisse + pilon). Ces phénomènes sont illustrés dans le tableau 9 à propos de 2 générations de nos lignées expérimentales. Les différences ne sont pas spectaculaires et pourraient être sans doute accrues encore par une sélection spécifique sur ces caractères; en effet les héritabilités mesurées par Ricard et Rouvier (1967 ; 1969) laissent supposer qu'une telle sélection spécifique sur le développement plus important des muscles chez la lignée maigre est probablement en partie le reflet de l'orientation particulière du métabolisme de ces animaux vers la protéinogénèse plutôt que vers la lipogenèse. Des observations similaires ont été réalisées sur les lignées expérimentales israéliennes et hollandaises (tableau 10). Il s'agit donc d'une retombée intéressante de la sélection contre l'engraissement à l'heure où les volailles sont de plus en plus vendues en parties découpées.
Des tests organoleptiques comparatifs ont été réalisés en France et en Hollande sur les génotypes maigres ou gras et n'ont révélé aucun défaut particulier du génotype maigre. A l'échelle du laboratoire (nombre limité de lignées comparées et de sujets testés) il semble donc que ce type de sélection n'ait guère de répercussion sur le goût et la texture des viandes.

\section{Autres conséquences de la sélection pour la maigreur}

L'évolution d'autres caractères au cours des sélections expérimentales a fait l'objet d'observations moins systématiques et parfois purement fortuites. On n'est d'ailleurs jamais sûr que les caractères aussi observés ne soient pas dus au hasard, les effectifs des lignées en sélection étant tout de même assez réduits, souvent 12 à 15 pères et 40 à 60 mères par lignée.

Signalons tout d'abord que chez les lignées sélectionnées à l'INRA les poussins maigres éclosent plus tôt que les poussins gras (Bernarczyk et al 1984). Aucune investigation n'a été entreprise pour tenter d'expliquer ce phénomène.

Les animaux maigres hollandais présentent moins de dyschondroplasie (troubles locomoteurs) que les animaux non sélectionnés. Ils sont également plus actifs (moins léthargiques). Cette dernière constatation n'a toutefois pas fait l'objet de tests quantitatifs.

Chez les adultes, les aptitudes à la reproduction ont été comparées. Dans les lignées INRA, la maturité sexuelle est identique et le nombre d'œufs n'est pas modifié. Seul le poids de l'œuf et sa composition subissent quelques change- 
ments significatifs. Les poules grasses, plus légères, pondent des oufs eux aussi plus légers, mais possédant une proportion de jaune nettement supérieure aux poules génétiquement maigres. Dans la sélection expérimentale réalisée en Ecosse, la lignée grasse nourrie à volonté présente des capacités de reproduction et une viabilité catastrophiques. Toutefois le rationnement efface les différences entre lignées et relève l'ensemble des paramètres (nombre d'œufs pondus, viabilité, fertilité, éclosivité). Cependant, comme en France, la lignée grasse pond des œufs plus petits. Au cours de l'expérience menée en Hollande, la sélection pour la réduction du gras abdominal ne modifie pas le nombre d'œufs pondus, alors que la sélection pour améliorer l'indice de consommation exerce un effet positif sur ce critère. La fertilité des mâles de la lignée sélectionnée pour un dépôt abdominal faible demeure équivalente à celle de la lignée témoin. En revanche, les coqs issus d'une sélection sur l'indice de consommation sont plus fertiles en reproduction naturelle. En insémination artificielle il est encore signalé une supériorité de la lignée sélectionnée sur l'indice de consommation.

Toutes ces observations tendent à montrer que les diverses méthodes envisagées pour réduire l'engraissement, soit ne modifient pas, soit améliorent légèrement les performances de reproduction. Aucune explication n'a été envisagée. En pratique, ces informations demeurent cependant très favorables à la sélection de croisement commerciaux maigres.

\section{Conclusion}

Les génotypes maigres, bien connus dans l'espèce porcine, peuvent constituer dans un avenir proche un progrès notoire en aviculture. Tous les agents des filières de production en tireront bénéfice. Pour le sélectionneur cela représente un effort méthodologique et technologique mais aussi un atout indéniable pour le maintien de sa compétitivité. Des économies substantielles sont prévisibles pour ce qui concerne l'énergie et les protéines alimentaires. En outre, il n'est pas interdit d'imaginer que la formulation des aliments soit renouvelée à l'occasion de l'apparition de ces nouveaux génotypes. On peut penser en effet que les normes d'apport en protéines et acides aminés d'une part, et en énergie d'autre part, subissent des modifications. En effet la faible adiposité peut contribuer, par exemple, à réduire les taux protéiques et à relever les taux énergétiques. Ces nouveaux génotypes pourraient très bien utiliser des régimes à " très haute énergie », très riches en matières grasses, sans manifester les excès d'engraissement que l'on rencontre avec les génotypes actuels dès que l'on veut élever le taux énergétique.

Les abattoirs valoriseront mieux ce type d'animal conçu pour son rendement élevé en parties de haute valeur commerciale et au contraire presque dépourvu en déchets gras. Enfin, le consommateur à la recherche de plats allégés pourra satisfaire ainsi à ses aspirations diététiques.

\section{Références bibliographiques}

BECKER W.A. SPENCER J.V., MIROSH L.W., VERSTRATE J A 1979. Prediction of fat-free live weight in broiler chickens using backskin fat, abdominal fat and live body weight. Poult. Sci, 58, 835-842.

BECKER W.A., SPENCER J.V., MIROSH L.W., VERSTRATE J.A., 1984. Genetic variation of abdominal fat, body weight and carcass weight in a female broiler line. Poult. Sci., 63, 607-611.

BERNARCZYK M.F., SIMON J., FERRE R., LECLERCQ B, 1984. Effets de la lumière sur le développement embryonnaire dans deux lignées de poulets rendus maigres ou gras par sélection. Reprod. Nutr. Develop., 24, 235-238.

BLUM J.C., LECLERCQ B., 1978. Influence de l'âge, du sexe et de l'alimentation sur la composition corporelle du pintadeau. In "Proceedings of the XVI world Poultry Congress », Rio de Janeiro, pp. 2029-2033.

CAHANER A., 1988. Experimental divergent selection on abdominal fat in broiler parental female and male type lines and their crosses. In « Leanness in domestic birds", p. 71-86. B. Leclercq et C.C. Whitehead Ed. - Butterworth, Grande-Bretagne.

CAHANFR A., NITSAN Z., 1985. Evaluation of simultaneous selection for live weight and against abdominal fat in broilers. Poult. Sci., 64, 1257-1263.

CAHANER A., NITSAN Z., 1986. Weight and fat content of adipose and non-adipose tissues in broilers selected for or against abdominal adipose tissue. Poult. Sci., 65 215-222.

CHAMBERS J.R., GAVORA J.S., 1982. Genetic parameters of broiler traits in synthetic parent populations. Poult. Sci., 61, 1434-1435

CHAMBERS J.R., BERNON D.E., GAVORA J.S., 1984 Synthesis and parameters of new populations of meattype chickens. Theor. Appl. Gen., 69, 23-30.

DEATON J.W., LOTT B.D., 1985. Age and dietary energy effect on broiler abdominal fat deposition. Poult. Sci., 64 2161-2164.

DELPECH P., RICARD F.H., 1965. Relation entre les dépôts adipeux viscéraux et les lipides corporels du poulet. Ann. Zootech., 14, 181-189.

EDWARDS HM, DENMAN F. ABOU-ASHOUR A., NUGARA D., 1975. Influence of age, sex and type of dietary fat supplementation on total carcass and fatty acid composition. Poult. Sci, 52, 934-948.

FRIARS G.W., LIN C.Y., PATTERSON D.L., IRWIN L.N., 1983. Genetic and phenotypic parameters of fat deposition and associated traits in broilers. Poult. Sci., 1245.

GERAERT P.A., GUILLAUMIN S., LECLERCQ B., LARBIER M., 1986. Utilisation des acides aminés à des fins énergétiques cher les poulets génétiquement maigres ou gras. In "Proceedings of the VII European Conference on Poultry Science », Paris, pp. 317-321.

GERAERT P.A., LECLERCQ B., LARBIER M., 1988a. Effects of dietary glucogenic amino acid supplementation on growth performance, body composition and plasma free amino acid levels in genetically lean or fat chickens Nutr. Reprod. Develop., 24, 1041-1051.

GERAERT P.A., MC LEOD M.G., LECLERCQ B., 1988b. Energy metabolism in genetically fat and lean chickens diet - and cold-induced thermogenesis. J. Nutr., 118 1232-1239.

GUILL R.A., WASHBURN K.W., 1974. Genetic changes in efficiency of feed utilization of chicks maintaining body weight constant. Poult. Sci., 53, 1146-1154.

GYLES N.R., MAEZA A., GOODWIN T.L., 1984. Regression of abdominal fat in broilers on abdominal fat in spent parents on severe feed restriction. Poult. Sci., 63, 1689-1694

KUBENA L.F. DEATON J.W., CHEN T.C., REECE F.N. 1974. Factors influencing the quantity of abdominal fat in broilers. Poult. Sci., 53, 211-214.

LECLERCQ B., 1983. The influence of dielary protein contents on the performances of genetically lean or fat growing chickens. Brit. Poult. Sci, 24, 581-587.

LECLERCQ B, 1988. Genetic selection of meat-type chickens for high or low abdominal fat content. In « Leanness in domestic birds \%, p. 25-40. B. Leclercq et C.C. Whilehead Ed. - Butterworth, Grande-Bretagne. 
LECLERCQ B., de CARVILLE H., 1978. Variabilité de quelques composants corporels chez le canard de Barbarie. In "Proceedings of the XVI World Poultry Congress ", Rio de Janeiro, pp. 2034-2036.

LECLERCQ B., ESCARTIN R., 1987. Further investigations on the effects of metabolisable energy content of diet on broiler performances. Archiv. Geflug., 51, 93-96.

LFCLERCQ B., GUY G., 1988. Plasma phospholipids and triglycerides in four experimental lines genetically different in their fattening. In "Leanness in domestic birds ", p. 235-237, B. Leclercq et C.C. Whitehead Ed., Butterworth, Grande-Bretagne.

LECLERCQ B., BLUM J.C., BOYER J.P., 1980. Selecting broilers for low or high abdominal fat: initial observations. Brit. Poult. Sci., 21, 107-113.

LECLERCQ. B., SIMON J., RICARD F.H., 1987. Effects of selection for high or low plasma glucose in chickens. Brit. Poult. Sci., 28, 557-565.

LECLERCQ B., GUY G., RUDEAUX F., 1989. Growth characteristics and lipid distribution in two lines of chickens selected for low or high abdominal fat. Génet. Sél. Evol., 21, 69-80.

LEENSTRA F.R., 1988. Selection for leanness : results of the Spelderholt experiment. In "Leanness in domestic birds » p. 59-69, B. Leclercq et C.C. Whitehead Ed., Butterworth, Grande-Bretagne.

LEENSTRA F.R., PIT R., 1987. Fat deposition in a broiler sire strain. II. Comparison amongst lines selected for less abdominal fat, lower feed conversion ratio, and higher body weight after restricted and ad-libitum feeding. Poult. Sci., 66, 193-202.

LEENSTRA F.R., PIT R., 1988. Consequences of selection for feed conversion in broiler chickens. In « Advances in animal breeding », p. 160-163, Wageningen.

LEENSTRA F.R., VEREIJKEN P.F.G., PIT R., 1986. Fat deposition in a broiler sire strain. I. Phenotypic and genetic variation in and correlations between, abdominal fat, body weight and feed conversion. Poult. Sci., 65, 1225-1235.

LEESON S., SUMMERS J.D., 1980. Production and carcass characteristics of the broiler chickens. Poult. Sci., 59, 786-798.

MALLARD J., DOUAIRE M., 1988. Strategies of selection for leanness in meat production. In "Leanness in domestic birds " p. 3-23. B. Leclercq et C.C. Whitehead Ed. Butterworth, Grande-Bretagne.

MAMMIER B., GERAERT P.A., LARBIER M., 1986. Metabolic utilization of leucine in a fat and a lean lines of chicken. Comp. Bioch. Physiol., 83A, 735-737.

PYM R.A., NICHOLLS P.J., 1979. Selection for food conversion in broilers : direct and correlated responses to selection for body-weight gain, food consumption and food conversion ratio. Brit. Poult. Sci., 20, 73-86.

PYM R.A., SOLVYNS A.J., 1979. Selection for food conversion in broilers: body composition of birds selected for increased body weight gain, food consumption and food conversion ratio. Brit. Poult. Sci., 20, 87-97.

PYM R.A., NICHOLLS P.J., THOMSON E., CHOICE A., FARRELL D.J., 1984. Energy and nitrogen metabolism of broilers selected over ten generations for increased growth rate, food intake and conversion of food to gain. Brit. Poult. Sci., 25, 529-539.
RICARD F.H., 1970. Etude d'un gène de nanisme lié au sexe chez la poule IV. Observations sur la croissance et les caractéristiques de carcasse du jeune poulet. Ann. Génét. Sél. anim., 2, 19-31.

RICARD F.H., 1978. Indice de consommation et état d'engraissement de poulets appartenant à des souches sélectionnées sur la forme de la courbe de croissance. In "Proceedings of the XVI World Poultry Congress ", Rio de Janeiro, pp. 1786-1798.

RICARD F.H., ROUVIER R., 1967. Etude de la composition anatomique du poulet. I. Variabilité de la répartition des différentes parties corporelles chez des coquelets "Bresse Pile». Ann. Zootech., 16, 23-29.

RICARD F.G., ROUVIER R., 1969. Etude la composition anatomique du poulet. II. Variabilité de la répartition des différentes parties corporelles dans une souche de type Cornish. Ann. Génét. Sél. anim., 1, 151-165.

RICARD F.H., TOURAILLE C., 1988. Selection for leanness and carcass quality. In "Leanness in domestic birds » p. 377-386, B. Leclercq et C.C. Whitehead Ed. Butterworth, Grande-Bretagne.

SIMON J.. ZYBKO A., GUILLAUME I., BLUM J.C., 1978. Recherche d'une limitation de l'engraissement du poulet de chair par un léger rationnement alimentaire entre 6 et 8 semaines. Arch. Geflug, 42, 6-9.

SORENSEN P., 1980. Selection for growth rate in broilers fed on diets with different protein levels in " Proceedings of the 6th European Poultry Conference ", Hamburg, pp. 64-71.

SORENSEN P., CHWALIBOG A., EGGUM B.O., 1983. Protein and energy metabolism in two lines chickens selected for growth on high or low protein diets. Brit. Poult. Sci., 24, 237-250.

THOMAS C.H., BLOW W.L., COCKERHAM C.C., GLAZENER E.W., 1958. The heritability of body weight, gain, feed consumption and feed conversion in broilers. Poult. Sci., $37,862-869$.

VAN MIDDELKOOP J.H., KUIT A.R., ZEGWERD A., 1976. Genetic factors in broiler fat deposition. In « Proceedings of the 12th Poultry Science Symposium. Growth and poultry meat production ", Edimburgh, pp. 131-143.

WHITEHEAD C.C., 1988. Selection for leanness in broilers using plasma lipoprotein concentration as selection criterion. In «Leanness in domestic birds », p. 41-57. B. Leclercq et C.C. Whitehead Ed. - Butterworth, GrandeBretagne.

WHITEHEAD C.C., GRIFFIN H.D., 1982. Plasma lipoprotein concentration as an indicator to fatness in broilers: effects of age and diet. Brit. Poult. Sci., 23, 299-305.

WHITEHEAD C.C., GRIFFIN H.D., 1984. Developpement of divergent lines of lean and fat broilers using plasma very low density lipoprotein concentration as selection criterion : the first three generations. Brit. Poult. Sci., 25, 573-582.

WILSON S.P., 1969. Genetic aspects of feed efficiency in broilers. Poult. Sci., 48, 487-495. 